\title{
OS BENEFÍCIOS DA TECNOLOGIA EMBARCADA EM IMPLEMENTOS AGRÍCOLAS PARA A AGRICULTURA DE PRECISÃO: estudo de caso de produção
} de uma indústria de implementos agrícolas

\section{THE BENEFITS OF TECHNOLOGY BOUNDED IN AGRICULTURAL IMPLEMENTS \\ FOR PRECISION AGRICULTURE: a case study of the production of an agricultural implements industry}

Jonatas William de Oliveira - jonataswilliam.oliveira@gmail.com
Carlos Rodrigo Volante - crvolante@hotmail.com Faculdade de Tecnologia de Taquaritinga (FATEC) -SP -Brasil

DOI: 10.31510/infa.v16i2.633

\section{RESUMO}

O objetivo deste artigo é apresentar os benefícios da tecnologia embarcada em implementos agrícolas para a agricultura de precisão, onde através da pesquisa bibliográfica, foi apresentado a evolução tecnológica dos implementos, o funcionamento da Agricultura de Precisão em campo, com suas tecnologias que promovem o ganho de produtividade gerando redução nos impactos ambientais e as possíveis tecnologias futuras que poderão ser utilizadas em implementos. Para se entender melhor o contex to abordado e apresentar os benefícios e as dificuldades que a indústria de implementos tendem a enfrentar, foi utilizado estudo de caso, onde foi possível se obter dados de uso no campo e se entender quais as dificuldades da empresa e do produtor para se atingir o lucro esperado dessa tecnologia. O trabalho conclui que a Agricultura de Precisão é um desafio muito grande para o produtor como para a indústria, visto que ainda são tecnologias caras e que exigem uma mão de obra qualificada, mas nada impossível de se resolver, conforme apresentado no decorrer deste artigo.

Palavras-chave: Agricultura. Precisão. Tecnologia. Benefícios.

\begin{abstract}
The aim of this paper is to present the benefits of embedded technology in agricultural implements for precision agriculture, where through bibliographic research, will be presented the technological evolution of implements, the operation of precision agriculture in the field, with its technologies that promote the gain. Productivity, reducing environmental impacts and the possible future technologies that could be used in implements. To better understand the context and to present the benefits and difficulties that the implement industry tends to face, a case study was used, where it was possible to obtain field use data and to understand the difficulties of the company and the producer. To achieve the expected profit of this technology. The paper concludes that Precision Farming is a major challenge for both
\end{abstract}




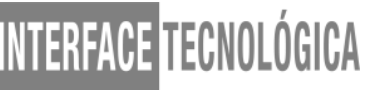

producer and industry, as they are still expensive technologies and require skilled labor, but nothing impossible to solve, as presented throughout this article.

Keywords: Agriculture. Precision. Technology. Benefits.

\section{INTRODUÇÃO}

A agricultura de precisão teve seu início na gestão da variabilidade espacial das lavouras, com o objetivo de melhorar a produtividade no campo, utilizando-se de tecnologias capazes de monitorar e gerenciar a adubação das lavouras com base em amostragem em solo georreferenciada (MOLIN, 2017).

De acordo com o mesmo autor, "a agricultura de precisão é um sistema de gerenciamento agrícola baseado na variação espacial de propriedades do solo e das plantas encontradas nas lavouras", a partir disso é possível se obter lucro, sustentabilidade e proteção do solo.

O principal objetivo desse artigo é apresentar os benefícios da tecnologia embarcada em implementos agrícolas para a agricultura de precisão, esclarecendo como as empresas de implementos agrícolas estão se comportando com as novas tecnologias e quais as vantagens que se obteve em campo, identificando possíveis dificuldades em seus implementos para suprir a nova demanda do mercado. Para isso será apresentado também a evolução dos implementos agrícolas, a fim de se compreender como surgiram as primeiras tecnologias em campo.

Com o objetivo de conhecer o novo modelo de implementos no mercado e suas tecnologias este trabalho se justifica por conta das inovações tecnológicas na agricultura, na qual as organizações buscam melhorar seus produtos para se estabelecer e ganhar um espaço no mercado, além de aperfeiçoar o manejo do solo com as novas tecnologias de precisão que vem ganhando espaço no mercado, tornando se um método de grande eficiência para o produtor. 


\section{A EVOLUÇÃO TECNOLÓGICA DOS IMPLEMENTOS AGRÍCOLAS}

Com o passar dos anos a indústria de implementos agrícolas passou por várias mudanças tecnológicas, buscando sempre aperfeiçoar e melhorar o trabalho no campo, de maneira mais sustentável a fim de se obter lucro, rapidez e proteção do solo.

Muzilli (1999) ressalta que, os sistemas agrícolas são tão antigos quanto mesmo a agricultura, onde há sido fabricada cerca de 4000 anos A.C., nessa época surgiu o primeiro arado inventado pelos egípcios.

Por conseguinte, a evolução das técnicas agrícolas determinou a diversificação progressiva dos implementos, e o metal substituiu a madeira. Com o descobrimento de novas formas de energia, como o vapor, a combustão e a eletricidade surgiram as máquinas agrícolas: tratores e colheitadeiras que aumentaram consideravelmente a produtividade rural e transformaram as condições de trabalho no campo (PROFETA FILHO; DEFFUNE PROFETA, 2009).

Segundo ABIMAQ (2006), ao passar dos anos, o ramo de capital mecânico vem se aperfeiçoando com investimentos constantes em questão da modernização do parque produtivo, visto que além de se aumentar a capacidade de produção, os recursos contribuem para o desenvolvimento tecnológico, visando a melhoria da qualidade dos produtos.

Segundo as definições de Schumpeter (1982), a inovação consiste nas novas formas de combinar fatores de produção e a essência está na novidade, sendo que ao empresário não inovador, resta a imitação. A inovação recém criada e difundida gera lucro extraordinário, e esse vai diminuindo à medida que vai sendo imitada, posteriormente.

Para Saenz e García Capote (2002), as tecnologias, dependendo da fase ou do momento em que são aplicadas, podem ser classificadas da seguinte forma: "Tecnologia de produto, Tecnologia de processo, Tecnologia de distribuição, Tecnologia de consumo, Tecnologia de gerência ou gestão, e, Tecnologia social” (2002, p. 50 - 51). Com isso, para a tecnologia em implementos são de utilidade a gestão e gerência, onde será possível controlar o mapeamento agrícola, sabendo onde será possível trabalhar com determinado implemento, na qual é feito desde o preparo do solo até a colheita do produto. 


\subsection{Características e vantagens da Agricultura de Precisão}

Segundo relata Abastecimento (2009), a Agricultura de Precisão tem como um dos seus principais objetivos oferecer o máximo aproveitamento das lavouras, utilizando se de tecnologias e estratégias a fim de se resolver problemas das lavouras que não são uniformes.

Hoje, especialmente no Brasil, as soluções existentes estão focadas na
aplicação de fertilizantes e corretivos em taxa variável, porém não se
deve perder de vista que AP é um sistema de gestão que considera a
variabilidade espacial das lavouras em todos seus aspectos:
produtividade, solo (características físicas, químicas, compactação
etc), infestação de ervas daninhas, doenças e pragas. (ABASTECIMENTO, 2009)

De acordo com Diego (2017), graças a Agricultura de Precisão é possível se obter mais informações técnicas, na qual antes não era possível se ter. Hoje a fim de se melhorar a produtividade no campo são utilizadas tecnologias, como piloto automático, aplicação de fertilizantes defensivos e/ou corretivos em taxa variável, além de se ter um melhor controle de pragas.

Com as novas necessidades de eficiência no setor agronómico para se manter nesse mercado competitivo, veio as novas tecnologias em geoprocessamento, sistemas de posicionamento global e muitas outras tecnologias que proporcionam a agricultura maior precisão e uma nova maneira de se enxergar as lavouras com características específicas. Segundo diz Fartogis (1998), "a agricultura de precisão promete reverter o quadro atual permitindo a aplicação de insumos agrícolas nos locais corretos e nas quantidades requeridas".

Segundo Davis (1998), agricultura de precisão é feita através da combinação de novas tecnologias na qual sustentam a era da informação com uma agricultura industrial madura. É um sistema de manejo de produção integrado, que tenta igualar o tipo e a quantia de insumos que entram na propriedade com as necessidades da cultura em pequenas áreas dentro de um campo da propriedade. Esta meta não é nova, mas novas tecnologias agora disponíveis permitem o conceito de agricultura de precisão ser percebido em uma produção prática. 


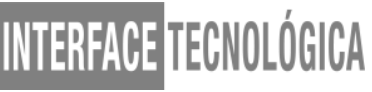

A partir do que abordado, é possível utilizar esse sistema como uma ferramenta técnica a fim de se resolver os problemas de desigualdade nas lavouras. Como um exemplo prático exposto por Diego (2017), a mesma quantidade de adubo era aplicada em toda área produzida, sem que as diferenças químicas e físicas do solo fossem consideradas. Com as tecnologias de precisão, o agricultor pode aplicar adubo de acordo com cada tipo de solo, que não é uniforme. Assim, ele economiza no uso de adubo e garante maior produtividade, por ter contemplado daquilo que o solo realmente precisava.

\subsection{Aplicações Tecnológicas Futuras para Implementos Agrícolas}

Atualmente o mundo está diante de inovações tecnológicas, onde essas devem se adequar ao homem e ao meio ambiente. Conforme explica Nelson e Winter (2005), a inovação e a invenção tem aspectos distintos, na qual com esses dois aspectos é possível se analisar possíveis tecnologias futuras. A inovação tem como foco a produção de um bem ou serviço por uma firma a fim de que seja comercializada e utilizada pelos consumidores, sendo que neste caso, tanto o fabricante, quanto o consumidor são inovadores. A invenção, por sua vez, nem sempre é passível de ser produzida e comercializada.

De acordo com Kline e Rosemberg (1986), o processo de desenvolvimento de uma inovação começa a partir da percepção de um problema ou de uma necessidade. A partir disso, atividades de pesquisa e desenvolvimento começam a ser realizadas a fim de se obter uma solução para tal. Entretanto, em alguns casos, a percepção pode ser em relação a um problema futuro, previamente reconhecido.

Segundo Rocha (1996, p.12-14), a tecnologia é o resultado de uma soma ciência e técnica, incorporando-se tanto a aplicação prática ou a apropriação econômica, quanto a sistemática do conhecimento científico. "É mais que um conjunto de produtos, processos e máquinas", "há também informações que organizam o saber fazer - uma aprendizagem que extrapola os bens e os meios utilizados para produzi-los”.

A partir do que foi visto, o cenário futurístico na qual robôs fazem todo o trabalho braçal, já chegou à produção agrícola. Protótipos de tratores, colheitadeiras e arados que podem ser comandados a distância já foram lançados no mercado. Nessas máquinas, o operador é substituído por sistemas inteligentes, sensores, rádio, dados e GPS que respondem 


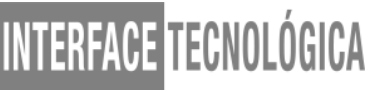

a comandos previamente configurados e são controlados por tablets e smartphones. A tecnologia está sendo considerada como uma evolução da agricultura de precisão.

\section{PROCEDIMENTOS METODOLÓGICOS}

Para identificar os benefícios da tecnologia embarcada em implementos agrícolas foi utilizado da pesquisa bibliográfica, na qual segundo Fonseca (2002), é feita através de levantamento de referências teóricas já analisadas, e publicadas por meio eletrônicos, como livros, artigos científicos e páginas de web sites. Os trabalhos científicos, costumam se iniciar através de uma pesquisa bibliográfica, onde é permitido ao pesquisador conhecer o que já se estudou sobre o assunto. A pesquisa bibliográfica tem como objetivo buscar respostas para solucionar o problema de pesquisa, na qual o pesquisador terá sobre seu conhecimento informações prévias a fim de se conhecer o problema a qual procura resposta.

Além disso, para melhor entender e identificar o problema de pesquisa, foi utilizado estudo de caso, que consiste na pesquisa de dados qualitativos, coletados a partir de eventos reais com o objetivo de explorar, explicar ou descrever, experiências e fenômenos atuais inseridos em seu próprio contexto. (Eisenhardt, 1989; Yin, 2009).

Para Yin (2009), o estudo de caso é útil para investigar novos conceitos, bem como para verificar como são aplicados e utilizados na prática elementos de uma teoria.

\section{RESULTADOS E DISCUSSÕES}

A presente análise foi realizada com base na comparação de dados coletados em campo apresentados pela revista engenharia agrícola e uma indústria de implementos agrícolas.

Com o surgimento de novas tecnologias em campo, as empresas produtoras de implementos agrícolas devem estudar e criar novos produtos para suprir essa demanda, na qual não é uma tarefa fácil, pois muitas das empresas brasileiras não estão com seus parques industriais preparados para esse novo tipo de produção. Para isso é fundamental a coleta de dados extraídos da produção no campo, apresentando informações com o uso da AP, comparando-se com os métodos antigos, na qual não havia nenhuma tecnologia de precisão. 
Na figura 1, demonstra os tipos de tecnologia da Agricultura de Precisão e qual a sua taxa de adoção pelos produtores. Com isso, são várias as tecnologias e as maneiras de utilizar as mesmas, na qual tudo depende da necessidade do agricultor, além disso, para a empresa atingir um bom nível de qualidade do produto e garantir a satisfação de seu cliente, ela deve analisar dados de campo e quais são as tecnologias mais utilizadas.

Figura 1: Taxa de adoção (\%) das tecnologias de agricultura de precisão pelos produtores pesquisados na região sudoeste de Goiás.

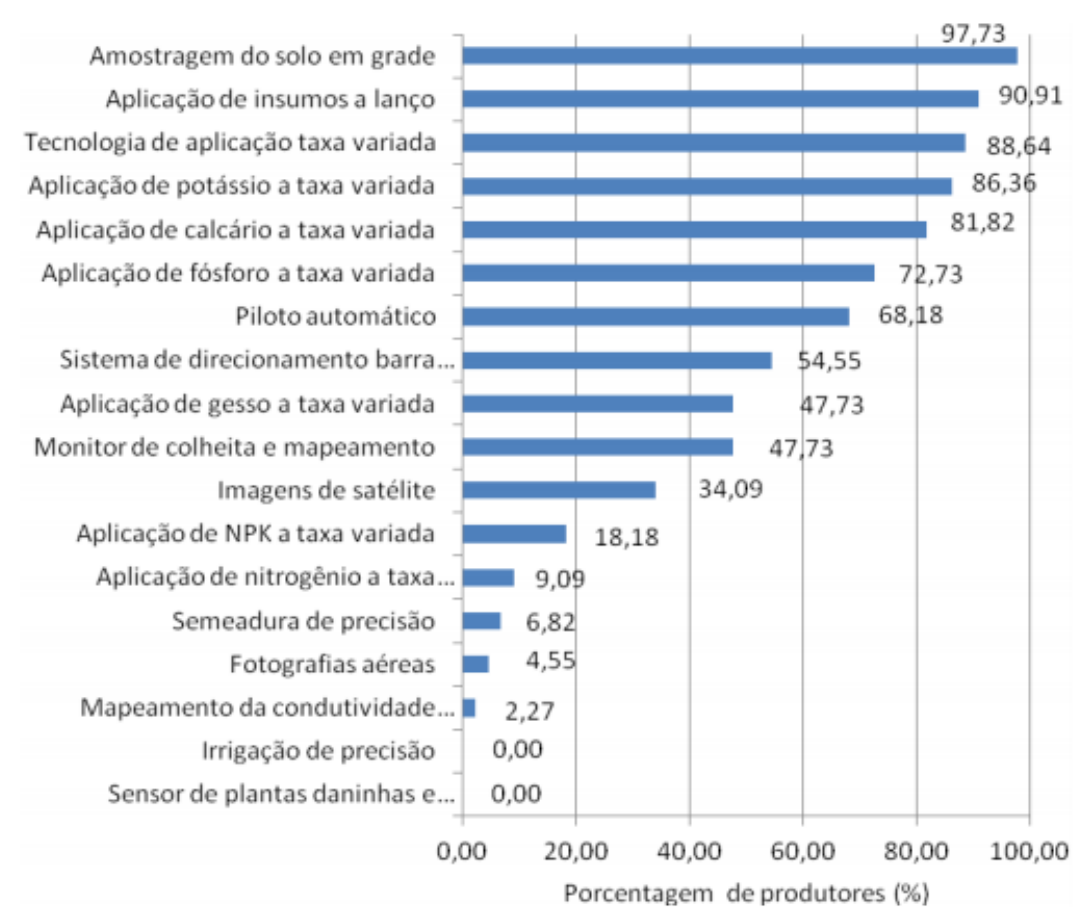

Fonte: http://dx.doi.org/10.1590/1809-4430-Eng.Agric.v35n4p689-698/2015

Com relação aos desafios em que a organização passa, estão eles representados na figura 2, na qual verificasse a falta de operadores e funcionários qualificados para a prestação de serviços além de se ter um alto custo da tecnologia da AP. Segundo relata Coelho, (2008) os elevados custos da tecnologia tendem a abaixar com o passar do tempo, já que o número de produtores que venham a adotar essas técnicas está crescendo cada vez mais, aliado a rápida evolução tecnológica.

Relacionado a falta de mão de obra qualificada para atuar na área, podem ser solucionadas através de parcerias com instituições de ensino, pesquisa e extensão rural, com o 
objetivo de qualificar pessoal, haja vista a complexidade da atividade. A aquisição de novas máquinas e equipamentos, considerada por 40,91\% dos produtores entrevistados como obstáculo de alta importância na adoção das tecnologias da AP, ocorre pelo custo de aquisição e de manutenção, além da necessidade da compatibilidade entre as diversas máquinas e equipamentos da propriedade.

Figura 2: Problemas e obstáculos na adoção da agricultura de precisão considerados de alta importância pelos produtores (\%) da região sudoeste de Goiás.

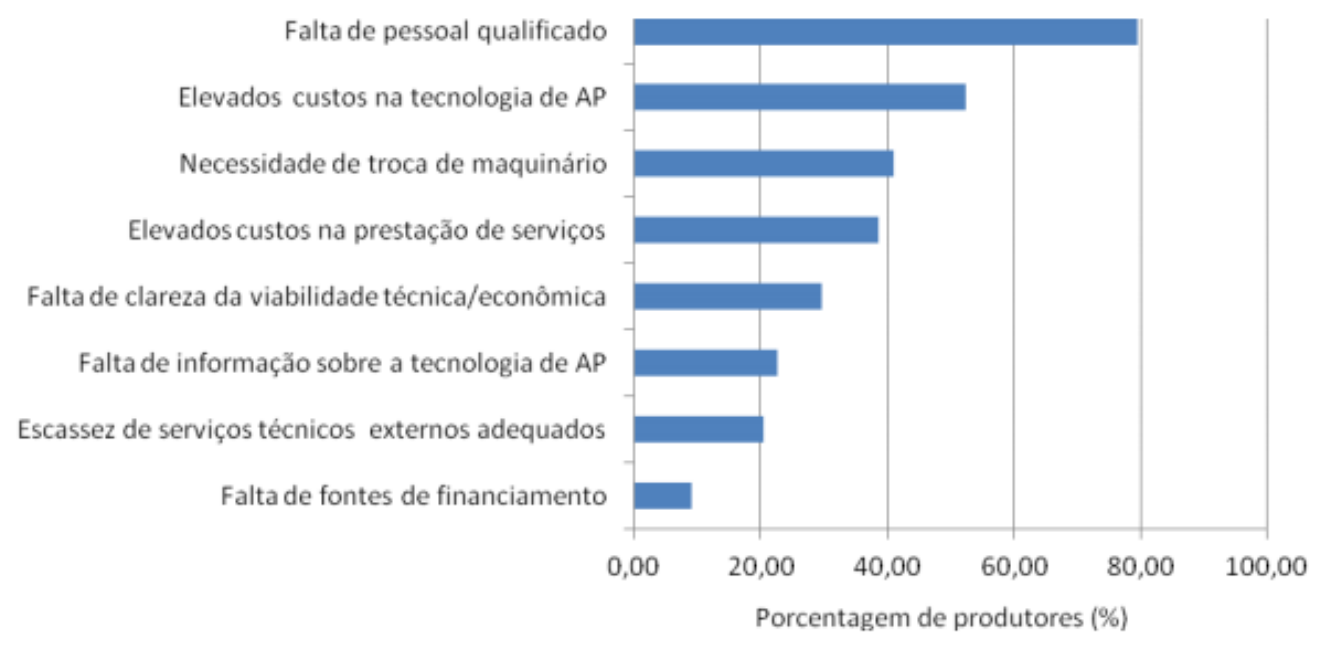

Fonte: http://dx.doi.org/10.1590/1809-4430-Eng.Agric.v35n4p689-698/2015

Em relação a qualificação da mão de obra para utilizar dos métodos da Agricultura de Precisão, cerca de $56,82 \%$ dos produtores disseram que não foi realizado treinamento para a prática desse novo meio de trabalho. O restante que representa 43,18\% dos produtores, afirmam que foram feitos treinamentos específicos para a prática da $\mathrm{AP}$, onde normalmente esses treinamentos são oferecidos pela empresa produtora desses implementos que venham a utilizar de tecnologias para a Agricultura de Precisão.

De acordo com entrevistados, a AP gera aumento na produtividade sendo considerado um impacto grande ou médio por 70,23\% dos entrevistados, mas ao se tratar de redução nos custos de produção, para $50 \%$ dos produtores, esse impacto é pequeno ou quase nulo, pois a diminuição do uso de insumos em uma gleba é compensada com o aumento da quantidade necessária em outras glebas, mantendo os custos constantes. 


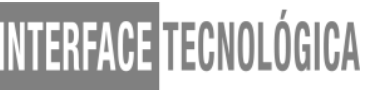

Para impactos ambientais, segundo 68,18\% dos produtores entrevistados a AP reduz impactos ambientais com o uso mais racional de insumos.

Visto que ao se analisar todas as informações obtidas em campo, para a empresa produtora de implementos agrícolas existe uma margem de lucro que varia de $40 \%$ a 56\%, lembrando que se concede descontos até $7 \%$ para revendas fieis.

\section{CONCLUSÃO}

Este artigo teve como objetivo apresentar os benefícios das tecnologias da Agricultura de Precisão utilizadas em implementos agrícolas, na qual para se entender melhor como funciona esse mundo e o que foi necessário para as empresas chegarem a esse novo conceito de agricultura, foi utilizado da pesquisa bibliográfica. A partir das pesquisas realizadas foi possível se chegar ao entendimento de que para a empresa de implementos agrícolas é um grande desafio se obter sucesso nesse mercado, visto que existem competições nessa área, onde quem conseguir criar produtos que atinjam as necessidades do produtor ganha um espaço no mercado.

Além disso, foi possível obter dados de campo através de entrevista com produtores de Goiás e juntamente com o estudo da pesquisa bibliográfica pode se concluir que, a AP tem vários benefícios para o produtor, onde ele pode utilizar das tecnologias para se obter um ganho na produtividade evitando impactos ambientais com a distribuição de insumos em taxa variável nos locais corretos e nas quantidades requeridas.

Para concluir este artigo o objetivo proposto foi esclarecer quais os benefícios da AP tanto para o produtor, como para as empresa produtora de implementos agrícolas, em que foi possível se entender que para as duas partes tem seus desafios para se utilizar desses métodos, como na falta de qualificação da mão de obra e nos altos custos de produção para a empresa por se tratar de uma tecnologia que nos dias de hoje são consideradas caras. Mas são de grandes vantagens para ambos os lados, visto que para a empresa a margem de lucro varia de $40 \%$ a $56 \%$ e para os produtores é possível se obter um aumento na produtividade no campo.

\section{REFERÊNCIAS}

ABASTECIMENTO, Ministério da Agricultura Pecuária e. Agricultura de Precisão

Boletim Técnico. Brasília: Assessoria de Comunicação Social, 2009. 31 p. Disponível em: 


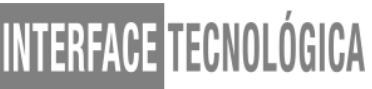

$<$ http://www.agricultura.gov.br/assuntos/insumos-agropecuarios/arquivos-publicacoesinsumos/agric_precisao.pdf>. Acesso em: 10 mai. 2019.

ABIMAQ. Associação Brasileira da Indústria de Máquinas e Equipamentos. Anuário ABIMAQ 2005. 2005. Disponível em: Acesso em: 01 jun. 2019.

CASTRO FILHO, C. de; MUZILLI, O. (Ed.). Uso e manejo dos solos de baixa aptidão agrícola. Londrina: IAPAR, 1999. 268 p.

COELHO,A. M. Agricultura de precisão em sistemas agrícolas. In: FALEIRO, F.G.; FARIAS NETO, A.L. Savanas: desafios e estratégias para o equilíbrio entre sociedade, agronegócio e recursos naturais. Planaltina, DF: Embrapa Cerrados, 2008. p. 1063-1083. Disponível em: Acesso em: 11 jul. 2019.

DIEGO, Rafael. Afinal, qual é a vantagem da agricultura de precisão? 2017. Disponível em: <http://www.senar.org.br/agricultura-precisao/afinal-qual-e-a-vantagem-da-agriculturade-precisao/>. Acesso em: 02 jun. 2019.

KLINE, S.; ROSENBERG, N. An overview of innovation. In.: LANDAU. R; ROSENBERG, N. (orgs). The positive strategy, Washington, DC: National Academy of Press, 1986.

NELSON, R. R.; WINTER, S. G. Uma teoria evolucionária da mudança econômica. Editora UNICAMP, 2005.

PROFETA FILHO, A. M.; DEFFUNE PROFETA, J. Revolução técnico-científica e globalização da economia. Fevereiro de 2009. Disponível em: Acesso em: 7 jan. 2019. ROCHA, IVAN. Ciência, tecnologia e inovação: conceitos básicos. Brasília: SEBRAE, 1996.

SAENZ, Tirso W.; GARCÍA CAPOTE, Emílio. Ciência, inovação e gestão tecnológica. Brasília: CNI/IEL/SENAI, ABIPTI, 2002.

SCHUMPETER, J. A. Teoria do desenvolvimento econômico: uma investigação sobre lucros, capital, crédito, juro e o ciclo econômico. São Paulo: Abril Cultural, 1982. (Série Os Economistas). 